\title{
Hardy-Type Inequalities on Time Scale via Convexity in Several Variables
}

\author{
Tzanko Donchev, ${ }^{1,2}$ Ammara Nosheen, ${ }^{2}$ and Josip Pečarić, ${ }^{2,3}$ \\ ${ }^{1}$ Department of Mathematics, University Al. I. Cuza, 700506 Iaşi, Romania \\ ${ }^{2}$ Abdus Salam School of Mathematical Sciences, Government College University, Lahore 54600, Pakistan \\ ${ }^{3}$ University of Zagreb, Faculty of Textile Technology, 10000 Zagreb, Croatia
}

Correspondence should be addressed to Josip Pečarić; pecaric@element.hr

Received 3 June 2013; Accepted 1 July 2013

Academic Editors: M. Lindstrom and S. Liu

Copyright (C) 2013 Tzanko Donchev et al. This is an open access article distributed under the Creative Commons Attribution License, which permits unrestricted use, distribution, and reproduction in any medium, provided the original work is properly cited.

We extend some Hardy-type inequalities with general kernels to arbitrary time scales using multivariable convex functions. Some classical and new inequalities are deduced seeking applications.

\section{Introduction}

The significant Hardy inequality is published in [1] (1952) (both in the continuous and discrete settings). More general Hardy integral inequalities have been studied in continuous cases. We notice only [2-6] and the references therein.

Recently, this inequality is studied in discrete case, and some variants of it are proved in case of time scales [7-9].

In [10], the authors study Hardy-type inequalities using convex functions of one variable with general kernels to arbitrary time scales.

The aim of this paper is to provide Hardy-type inequalities using multivariable convex function with general kernels to arbitrary time scales. Notice that time scales include continuous and discrete time cases under unified approach.

Firstly, we recall necessary preliminary facts needed afterward. The main results are given in Section 3. Section 4 is devoted to some inequalities with certain kernels. In the last section, we discuss some particular cases of Hardy-type inequalities.

\section{Preliminaries}

First, we recall the basic concepts used in the paper and refer the interested reader to [11] for the theory of time scales.
A time scale is any nonempty closed subset of the real line $\mathbb{T} \subset \mathbb{R}$. On nondensity points we define forward, respectively, backward jump operators $\sigma, \rho: \mathbb{T} \rightarrow \mathbb{T}$ as

$$
\begin{aligned}
& \sigma(t)=\inf \{s \in \mathbb{T}: s>t\}, \\
& \rho(t)=\sup \{s \in \mathbb{T}: s<t\} .
\end{aligned}
$$

The point $t$ is said to be right-scattered if $\sigma(t)>t$ and leftscattered if $\rho(t)<t$, respectively. Clearly, $t$ is right-dense if $\sigma(t)=t$ and left dense if $\rho(t)=t$, respectively.

Let $n \in \mathbb{N}$; we define $n$-dimensional time scale by the Cartesian product of given time scales $\mathbb{T}_{i}, i \in\{1, \ldots, n\}$, as

$$
\Omega^{n}=\left\{\vec{a}=\left(a_{1}, a_{2}, \ldots, a_{n}\right): a_{i} \in \mathbb{T}_{i}, i \in\{1, \ldots, n\}\right\} .
$$

Evidently, $\Omega^{n}$ is a complete metric space with distance as follows:

$$
d(\vec{a}, \vec{b})=\left(\sum_{i=1}^{n}\left|b_{i}-a_{i}\right|^{2}\right)^{1 / 2}, \quad a, b \in \Omega^{n} .
$$

Now we are going to describe the construction of Lebesgue measure in $\Omega^{n}$. We refer to [12-14] for the theory of measure spaces and measurable functions on time scales.

Let $\mathscr{F}$ be the family of all $n$-dimensional time scale intervals in $\Omega^{n}$; that is,

$$
V=[\vec{a}, \vec{b})=\left[a_{1}, b_{1}\right) \times\left[a_{2}, b_{2}\right) \times \cdots \times\left[a_{n}, b_{n}\right),
$$


with $\vec{a}=\left(a_{1}, a_{2}, \ldots, a_{n}\right), \vec{b}=\left(b_{1}, b_{2}, \ldots, b_{n}\right) \in \Omega^{n}$, and $a_{i} \leq b_{i}$ for all $i \in\{1, \ldots, n\}$. Let $m: \mathscr{F} \rightarrow[0, \infty)$ be the set function that assigns to each $n$-dimensional time scale interval $V=$ $[a, b)$ its volume as follows:

$$
m(V)=\prod_{i=1}^{n}\left(b_{i}-a_{i}\right) .
$$

Let $\emptyset \neq E \subset \Omega^{n}$. If there exists finite or countable system of pairwise disjoint $n$-dimensional time scale intervals $V_{k}=$ $\left[\vec{a}_{k}, \vec{b}_{k}\right)$ with $E \subset \bigcup_{k \geq 1} V_{k}$, then the outer measure $m^{*}$ of $E$ is defined by

$$
m^{*}(E)=\inf \left\{\sum_{k \geq 1} m\left(V_{k}\right), E \subset \bigcup_{k \geq 1} V_{k}\right\} .
$$

If there is no such covering of $E$, then $m^{*}(E)=\infty$.

A subset $A$ of $\Omega^{n}$ is said to be measurable (or $\Delta$ measurable) if

$$
m^{*}(E)=m^{*}(E \cap A)+m^{*}\left(E \cap A^{c}\right)
$$

holds for all $E \subset \Omega^{n}$, where $A^{c}=\Omega^{n} \backslash A$. The family $\mathscr{M}$ of all $m^{*}$-measurable subsets of $\Omega^{n}$ is a $\sigma$-algebra generated by $\mathscr{F}$. The restriction of $m^{*}$ to $\mathscr{M}$, which we denote by $\mu_{\Delta}$, is a $\sigma$-additive measure on $\mathscr{M}$. Clearly, $\mathscr{F} \subset \mathscr{M}$ and $\mu_{\Delta}(V)=m(V)$ for each $V \in \mathscr{F}$. The measure $\mu_{\Delta}$ (called the Lebesgue $\Delta$-measure on $\Omega^{n}$ ) is Carathéodory extension of the original measure $m$ defined on $\mathscr{F}$. We call $\left(\Omega^{n}, \mathscr{M}, \mu_{\Delta}\right)$ an $n$ dimensional time scale measure space.

Denote $\widetilde{a}_{i}=\inf _{t \in \mathbb{\mathbb { T }}_{i}} t$. Similarly, $\widetilde{b}_{i}=\sup _{t \in \mathbb{J}_{i}} t$. Let $\Omega_{\infty}^{n}$ be the set of all points $\vec{b}=\left(b_{1}, b_{2}, \ldots, b_{n}\right) \in \Omega^{n}$ for which there exists at least one $b_{i}$ such that $b_{i}=\widetilde{b}_{i}$. From [11, Theorem 3.1], we know that if $\vec{t}=\left(t_{1}, t_{2}, \ldots, t_{n}\right) \in \Omega^{n} \backslash \Omega_{\infty}^{n}$, then the singlepoint set $\{t\}$ is $\Delta$-measurable and

$$
\mu_{\Delta}(\{t\})=\prod_{i=1}^{n}\left(\sigma_{i}\left(t_{i}\right)-t_{i}\right) .
$$

Obviously, for all $i \in\{1, \ldots, n\}$, the set $\Omega_{0}^{n}=\Omega^{n} \backslash \Omega_{\infty}^{n}$ can be represented as a finite or countable union of intervals of the family $\mathscr{F}$; hence it is $\Delta$-measurable. Furthermore, the set $\Omega_{\infty}^{n}=\Omega^{n} \backslash \Omega_{0}^{n}$ is $\Delta$-measurable being the difference of two $\Delta$-measurable sets $\Omega^{n}$ and $\Omega_{0}^{n}$, but $\Omega_{\infty}^{n}$ does not have a finite or countable covering intervals of $\mathscr{F}$, that is, for any $\Delta$-measurable subset $A$ of $\Omega^{n}$ such that $A \cap \Omega_{\infty} \neq \emptyset$ has $\Delta$ measure $\infty$. In particular, if $a \in \mathbb{T}$, where $\mathbb{T}$ is an arbitrary time scale, then the set $[a, \infty)=\{t \in \mathbb{T}: a \leq t\}$ is $\Delta$ measurable.

The function $f: \Omega^{n} \rightarrow \overline{\mathbb{R}}:=[-\infty, \infty]$ is said to be $\Delta-$ measurable if for every $\alpha \in \mathbb{R}$, the set $f^{-1}([-\infty, \alpha))=\{t=$ $\left.\left(t_{1}, \ldots, t_{n}\right) \in \Omega^{n}: f(t)<\alpha\right\}$ is $\Delta$-measurable. It is easy to see that $f$ is $\Delta$-measurable if and only if for each open set $G \subset \mathbb{R}$, the set $f^{-1}(G)=\left\{t \in \Omega^{n}: f(t) \in G\right\}$ is $\Delta$-measurable. Moreover, if $f: \Omega^{n} \rightarrow \mathbb{R}$ is $\Delta$-measurable and $\Phi: I \subset \mathbb{R} \rightarrow \mathbb{R}$ is a continuous function, then $\Phi \circ f: \Omega^{n} \rightarrow \mathbb{R}$ is $\Delta$-measurable.

Having the $\sigma$-additive measure $\mu_{\Delta}$ on $\Omega^{n}$, we possess the corresponding integration theory for functions $f: E \subset$ $\Omega^{n} \rightarrow \mathbb{R}$, according to the general Lebesgue integration theory (see, e.g., [15]). The Lebesgue integral associated with the measure $\mu_{\Delta}$ on $\Omega^{n}$ is called the Lebesgue $\Delta$-integral. For a $\Delta$-measurable set $E \subset \Omega^{n}$ and a $\Delta$-measurable function $f: E \rightarrow \mathbb{R}$, the corresponding $\Delta$-integral of $f$ over $E$ will be denoted by

$$
\int_{E} f\left(t_{1}, \ldots, t_{n}\right) \Delta t_{1} \cdots \Delta t_{n} \quad \text { or } \quad \int_{E} f(t) \Delta t .
$$

Notice that all theorems of the general Lebesgue integration theory, including the Lebesgue dominated convergence theorem, hold also for Lebesgue $\Delta$-integrals on $\Omega^{n}$.

If $\mathbb{T}$ is a time scale and the interval $[a, b) \subset \mathbb{T}$ contains only isolated points, then

$$
\int_{a}^{b} f(t) \Delta t=\sum_{t \in[a, b)}(\sigma(t)-t) f(t) .
$$

Let $\left(\Omega, \mathscr{M}, \mu_{\Delta}\right)$ and $\left(\Lambda, \mathscr{L}, \lambda_{\Delta}\right)$ be two finite dimensional time scale measure spaces. We define the product measure space $\left(\Omega \times \Lambda, \mathscr{M} \times \mathscr{L}, \mu_{\Delta} \times \lambda_{\Delta}\right)$, where $\mathscr{M} \times \mathscr{L}$ is the product $\sigma$-algebra generated by $\{E \times F: E \in \mathscr{M}, F \in \mathscr{L}\}$ and

$$
\left(\mu_{\Delta} \times \lambda_{\Delta}\right)(E \times F)=\mu_{\Delta}(E) \lambda_{\Delta}(F) .
$$

Fubini's Theorem in Time Scales see ([16, Theorem 1.1]). If $f$ : $\Omega \times \Lambda \rightarrow \mathbb{R}$ is a $\mu_{\Delta} \times \lambda_{\Delta}$-integrable function and if we define the function $\varphi(y)=\int_{\Omega} f(x, y) \Delta x$ for a.e. $y \in \Lambda$ and $\psi(x)=$ $\int_{\Lambda} f(x, y) \Delta y$ for a.e. $x \in \Omega$, then $\varphi$ is $\lambda_{\Delta}$-integrable on $\Lambda, \psi$ is $\mu_{\Delta}$-integrable on $\Omega$, and

$$
\int_{\Omega} \Delta x \int_{\Lambda} f(x, y) \Delta y=\int_{\Lambda} \Delta y \int_{\Omega} f(x, y) \Delta x .
$$

\section{Inequalities with General Kernels}

Let $\mathbf{f}(y)=\left(f_{1}(y), \ldots, f_{m}(y)\right)$ be $m$-tuple of functions such that $f_{j}(y)$ are $\lambda_{\Delta}$-integrable for all $j \in\{1, \ldots, m\}$. Then $\int_{\Lambda} \mathbf{f}(y) \Delta(y)$ denotes the $m$-tuple $\left(\int_{\Lambda} f_{1}(y) \Delta(y), \ldots\right.$, $\left.\int_{\Lambda} f_{m}(y) \Delta(y)\right)$; that is, $\Delta$-integral acts on each component of $\mathbf{f}(y)$.

The following Jensen's inequality on time scales is given in [17, Theorem 2.1].

Theorem 1. Let $\left(\Omega, \mathscr{M}, \mu_{\Delta}\right)$ and $\left(\Lambda, \mathscr{L}, \lambda_{\Delta}\right)$ be two time scale measure spaces. Suppose $U \subset \mathbb{R}^{m}$ is a closed convex set, $\Phi \in$ $C(U, \mathbb{R})$ is convex, and $\mathbf{f}(\Lambda) \subset U$. Moreover, let $k: \Omega \times \Lambda \rightarrow \mathbb{R}$ be nonnegative such that $k(x, \cdot)$ is $\lambda_{\Delta}$-integrable. Then one has

$$
\Phi\left(\frac{\int_{\Lambda} k(x, y) \mathbf{f}(y) \Delta y}{\int_{\Lambda} k(x, y) \Delta y}\right) \leq \frac{\int_{\Lambda} k(x, y) \Phi(\mathbf{f}(y)) \Delta y}{\int_{\Lambda} k(x, y) \Delta y}
$$

Throughout this section, we assume that the following hypotheses hold.

H1 $\left(\Omega, \mathscr{M}, \mu_{\Delta}\right)$ and $\left(\Lambda, \mathscr{L}, \lambda_{\Delta}\right)$ are two time scale measure spaces.

$\mathbf{H} 2 k: \Omega \times \Lambda \rightarrow \mathbb{R}_{+}$is such that $K(x):=\int_{\Lambda} k(x, y) \Delta y<$ $\infty, x \in \Omega$.

$\mathbf{H 3} \xi: \Omega \rightarrow \mathbb{R}_{+}$is such that $w(y):=\int_{\Omega}(k(x, y) \xi(x) /$ $K(x)) \Delta x, y \in \Lambda$. 
Theorem 2. If $U \subset \mathbb{R}^{m}$ is a closed convex set such that $\Phi$ : $U \rightarrow \mathbb{R}$ is convex and continuous, then

$$
\begin{gathered}
\int_{\Omega} \xi(x) \Phi\left(\frac{1}{K(x)} \int_{\Lambda} k(x, y) \mathbf{f}(y) \Delta y\right) \Delta x \\
\leq \int_{\Lambda} w(y) \Phi(\mathbf{f}(y)) \Delta y
\end{gathered}
$$

holds for all $\lambda_{\Delta}$-integrable functions $\mathbf{f}: \Lambda \rightarrow \mathbb{R}^{m}$ such that $\mathbf{f}(\Lambda) \subset U$.

Proof. Using Jensen's inequality (13) for several variables and the Fubini theorem on time scales, we find that

$$
\begin{aligned}
\int_{\Omega} \xi(x) \Phi\left(\frac{1}{K(x)} \int_{\Lambda} k(x, y) \mathbf{f}(y) \Delta y\right) \Delta x \\
=\int_{\Omega} \xi(x) \Phi\left(\frac{1}{K(x)} \int_{\Lambda} k(x, y) f_{1}(y) \Delta y, \ldots, \frac{1}{K(x)}\right. \\
\left.\quad \times \int_{\Lambda} k(x, y) f_{m}(y) \Delta y\right) \Delta x \\
\leq \int_{\Omega} \frac{\xi(x)}{K(x)}\left(\int_{\Lambda} k(x, y) \Phi(\mathbf{f}(y)) \Delta y\right) \Delta x \\
=\int_{\Lambda} \Phi(\mathbf{f}(y))\left(\int_{\Omega} \frac{k(x, y) \xi(x)}{K(x)} \Delta x\right) \Delta y \\
=\int_{\Lambda} w(y) \Phi(\mathbf{f}(y)) \Delta y .
\end{aligned}
$$

The proof is therefore complete.

Remark 3. If $\Phi$ is concave, then (14) holds in reverse direction.

Corollary 4. Let $\Psi:\left[l_{1}, \hat{l}_{1}\right) \times \cdots \times\left[l_{m}, \hat{l}_{m}\right) \rightarrow \mathbb{R}_{+}$be continuous function and define

$$
\widetilde{L}_{j}\left(f_{j}, \Lambda\right)=L_{j}^{-1}\left(\frac{\int_{\Lambda} k(x, y) L_{j}\left(f_{j}(y)\right) \Delta y}{K(x)}\right)
$$

for all $j \in\{1, \ldots, m\}$. If $\Phi\left(s_{1}, \ldots, s_{m}\right)=\Psi\left(L_{1}^{-1}\left(s_{1}\right), \ldots\right.$, $\left.L_{m}^{-1}\left(s_{m}\right)\right)$ is convex, then

$$
\begin{array}{r}
\int_{\Omega} \xi(x) \Psi\left(\widetilde{L}_{1}\left(f_{1}, \Lambda\right), \ldots, \widetilde{L}_{m}\left(f_{m}, \Lambda\right)\right) \Delta x \\
\leq \int_{\Lambda} w(y) \Psi\left(f_{1}(y), \ldots, f_{m}(y)\right) \Delta y
\end{array}
$$

holds for all $f_{j}(\Lambda) \subset\left[l_{j}, \hat{l}_{j}\right)$ and continuous monotone functions $L_{j}:\left[l_{j}, l_{j}\right) \rightarrow \mathbb{R}$ such that $L_{j} \circ f_{j}$ are $\lambda_{\Delta}$-integrable for all $j \in\{1, \ldots, m\}$.

Proof. Replace in Theorem $2 f_{j}(y)$ with $L_{j}\left(f_{j}(y)\right)$ for all $j \in\{1, \ldots, m\}$ and $\Phi\left(s_{1}, \ldots, s_{m}\right)$ with $\Psi\left(L_{1}^{-1}\left(s_{1}\right), \ldots, L_{m}^{-1}\left(s_{m}\right)\right)$.
Remark 5. In case that $\mathbb{T}=\mathbb{N}$ and $m=2$, Corollary 4 is as [18, Corollary 1.2].

Remark 6. In case that $m=2$, we can use the results of Beck [19] (see also [20], page 194) as applications of Corollary 4, which corresponds to the generalizations of Hölder's and Minkoski's inequalities. In classical case, many authors have studied these types of generalizations; see, for example, [2123].

Furthermore, in the paper, we use $p>1$ with $(1 / p)+$ $(1 / \hat{p})=1$.

Corollary 7. If $\mathbf{f}(x)=\left(f_{1}(x), f_{2}(x)\right)$, then

$$
\begin{aligned}
\int_{\Omega} \xi(x) & \left(\frac{1}{K(x)} \int_{\Lambda} k(x, y)\left(f_{1}(y)\right)^{p} \Delta y\right)^{1 / p} \\
\times & \left(\frac{1}{K(x)} \int_{\Lambda} k(x, y)\left(f_{2}(y)\right)^{p} \Delta y\right)^{1 / p} \Delta x \\
& \geq \int_{\Lambda} w(y) f_{1}(y) f_{2}(y) \Delta y
\end{aligned}
$$

holds for all $\lambda_{\Delta}$-integrable $f_{j}: \Lambda \rightarrow \mathbb{R}_{+}$, where $j \in\{1,2\}$.

Proof. Use $m=2, \Psi\left(s_{1}, s_{2}\right)=s_{1} s_{2}, L_{1}\left(t_{1}\right)=\left(t_{1}\right)^{p}, L_{2}\left(t_{2}\right)=$ $\left(t_{2}\right)^{\dot{p}}$ in Corollary 4; then $\Phi\left(s_{1}, s_{2}\right)=s_{1}^{1 / p} s_{2}^{1 / \hat{p}}$ is concave in Theorem 2.

Corollary 8. If $\mathbf{f}(x)=\left(f_{1}(x), f_{2}(x)\right)$, then

$$
\begin{aligned}
& \int_{\Omega} \xi(x)\left(\left(\frac{1}{K(x)} \int_{\Lambda} k(x, y)\left(f_{1}(y)\right)^{p} \Delta y\right)^{1 / p}\right. \\
&\left.+\left(\frac{1}{K(x)} \int_{\Lambda} k(x, y)\left(f_{2}(y)\right)^{p} \Delta y\right)^{1 / p}\right)^{p} \Delta x \\
& \geq \int_{\Lambda} w(y)\left(f_{1}(y)+f_{2}(y)\right)^{p} \Delta y
\end{aligned}
$$

holds for all $\lambda_{\Delta}$ integrable $f_{j}: \Lambda \rightarrow \mathbb{R}_{+}$, where $j \in\{1,2\}$.

Proof. Use $m=2, \Psi\left(s_{1}, s_{2}\right)=\left(s_{1}+s_{2}\right)^{p}, L_{1}\left(t_{1}\right)=\left(t_{1}\right)^{p}$, $L_{2}\left(t_{2}\right)=\left(t_{2}\right)^{p}$ in Corollary 4 ; then $\Phi\left(s_{1}, s_{2}\right)=\left(s_{1}^{1 / p}+s_{2}^{1 / p}\right)^{p}$ is concave in Theorem 2 .

Remark 9. If $p<1$, then reverse inequalities hold in Corollaries 4, 7, and 8.

\section{Inequalities with Special Kernels}

Throughout this section and in the next section, we assume that the following hypothesis holds.

$\mathbf{H 4} \Omega=\Lambda=\left[a_{1}, b_{1}\right)_{\mathbb{T}} \times\left[a_{2}, b_{2}\right)_{\mathbb{T}} \times \cdots \times\left[a_{n}, b_{n}\right)_{\mathbb{T}}, 0 \leq a_{i}<$ $b_{i} \leq \infty$ for all $i \in\{1, \ldots, n\}$, where $\mathbb{T}$ is an arbitrary time scale. 
Corollary 10. Assume that

$$
\begin{aligned}
& \xi: \Omega \rightarrow \mathbb{R}_{+} \text {is such that } \\
& \begin{aligned}
w(y)=\int_{y_{1}}^{b_{1}} \cdots \int_{y_{n}}^{b_{n}} & \frac{k\left(x_{1}, \ldots, x_{n}, y\right) \xi\left(x_{1}, \ldots, x_{n}\right)}{K\left(x_{1}, \ldots, x_{n}\right)} \\
& \quad \times \Delta x_{1} \cdots \Delta x_{n}, \quad y \in \Lambda .
\end{aligned}
\end{aligned}
$$

If $U \subset \mathbb{R}^{m}$ is a closed convex set such that $\Phi: U \rightarrow \mathbb{R}$ is convex and continuous; then

$$
\begin{gathered}
\int_{a_{1}}^{b_{1}} \cdots \int_{a_{n}}^{b_{n}} \xi\left(x_{1}, \ldots, x_{n}\right) \Phi\left(\left(A_{k} \mathbf{f}\right)\left(x_{1}, \ldots, x_{n}\right)\right) \Delta x_{1} \cdots \Delta x_{n} \\
\leq \int_{a_{1}}^{b_{1}} \cdots \int_{a_{n}}^{b_{n}} w\left(y_{1}, \ldots, y_{n}\right) \Phi\left(\mathbf{f}\left(y_{1}, \ldots, y_{n}\right)\right) \\
\times \Delta y_{1} \cdots \Delta y_{n}
\end{gathered}
$$

holds for all $\lambda_{\Delta}$-integrable $\mathbf{f}: \Lambda \rightarrow \mathbb{R}^{m}$ such that $\mathbf{f}(\Lambda) \subset U$, where

$$
\begin{aligned}
\left(A_{k} \mathbf{f}\right)(x)=\frac{1}{K(x)} \int_{a_{1}}^{\sigma\left(x_{1}\right)} \cdots \int_{a_{n}}^{\sigma\left(x_{n}\right)} k\left(x, y_{1}, \ldots, y_{n}\right) & \\
& \quad \times \mathbf{f}\left(y_{1}, \ldots, y_{n}\right) \Delta y_{1} \cdots \Delta y_{n} .
\end{aligned}
$$

Proof. Statement follows from Theorem 2 using

$$
\begin{array}{r}
k\left(x_{1}, \ldots, x_{n}, y_{1}, \ldots, y_{n}\right)=0, \quad \text { if } a_{i} \leq y_{i} \leq \sigma\left(x_{i}\right) \\
\text { for all } i \in\{1, \ldots, n\},
\end{array}
$$

since in this case

$$
K(x)=\int_{a_{1}}^{\sigma\left(x_{1}\right)} \cdots \int_{a_{n}}^{\sigma\left(x_{n}\right)} k\left(x, y_{1}, \ldots, y_{n}\right) \Delta y_{1} \cdots \Delta y_{n} .
$$

\section{Corollary 11. Assume that}

$$
\xi: \Omega \longrightarrow \mathbb{R}_{+} \text {is such that }
$$

$$
\begin{gathered}
w(y)=\int_{a_{1}}^{y_{1}} \cdots \int_{a_{n}}^{y_{n}} \frac{k\left(x_{1}, \ldots, x_{n}, y\right) \xi\left(x_{1}, \ldots, x_{n}\right)}{K\left(x_{1}, \ldots, x_{n}\right)} \\
\times \Delta x_{1} \cdots \Delta x_{n}, \quad y \in \Lambda .
\end{gathered}
$$

If $U \subset \mathbb{R}^{m}$ is a closed convex set such that $\Phi: U \rightarrow \mathbb{R}$ is convex and continuous, then (21) holds for all $\lambda_{\Delta}$-integrable $\mathbf{f}$ : $\Lambda \rightarrow \mathbb{R}^{m}$ such that $\mathbf{f}(\Lambda) \subset U$, and

$$
\begin{aligned}
\left(A_{k} \mathbf{f}\right)(x)=\frac{1}{K(x)} \int_{\sigma\left(x_{1}\right)}^{b_{1}} \cdots \int_{\sigma\left(x_{n}\right)}^{b_{n}} k\left(x, y_{1}, \ldots, y_{n}\right) & \\
& \quad \times \mathbf{f}\left(y_{1}, \ldots, y_{n}\right) \Delta y_{1} \cdots \Delta y_{n} .
\end{aligned}
$$

Proof. Statement follows from Theorem 2 using

$$
\begin{array}{r}
k\left(x_{1}, \ldots, x_{n}, y_{1}, \ldots, y_{n}\right)=0, \quad \text { if } a_{i} \leq \sigma\left(x_{i}\right) \leq y_{i} \\
\text { for all } i \in\{1, \ldots, n\},
\end{array}
$$

since in this case

$$
K(x)=\int_{\sigma\left(x_{1}\right)}^{b_{1}} \ldots \int_{\sigma\left(x_{n}\right)}^{b_{n}} k\left(x, y_{1}, \ldots, y_{n}\right) \Delta y_{1} \cdots \Delta y_{n} .
$$

Theorem 12. Assume that

$\xi: \Omega \rightarrow \mathbb{R}_{+}$is such that

$$
\widetilde{w}(y)=\int_{y_{1}}^{b_{1}} \cdots \int_{y_{n}}^{b_{n}} \frac{\xi\left(x_{1}, \ldots, x_{n}\right)}{\prod_{i=1}^{n}\left(\sigma\left(x_{i}\right)-a_{i}\right)} \Delta x_{1} \cdots \Delta x_{n}, \quad y \in \Lambda .
$$

If $U \subset \mathbb{R}^{m}$ is a closed convex set such that $\Phi: U \rightarrow \mathbb{R}$ is convex and continuous, then

$$
\begin{gathered}
\int_{a_{1}}^{b_{1}} \ldots \int_{a_{n}}^{b_{n}} \xi\left(x_{1}, \ldots, x_{n}\right) \Phi\left(\left(\widetilde{A_{k}} \mathbf{f}\right)\left(x_{1}, \ldots, x_{n}\right)\right) \Delta x_{1} \cdots \Delta x_{n} \\
\leq \int_{a_{1}}^{b_{1}} \ldots \int_{a_{n}}^{b_{n}} \widetilde{w}\left(y_{1}, \ldots, y_{n}\right) \Phi\left(\mathbf{f}\left(y_{1}, \ldots, y_{n}\right)\right) \\
\times \Delta y_{1} \cdots \Delta y_{n}
\end{gathered}
$$

holds for all $\lambda_{\Delta}$-integrable $\mathbf{f}: \Lambda \rightarrow \mathbb{R}^{m}$ such that $\mathbf{f}(\Lambda) \subset U$, where

$$
\begin{aligned}
\left(\widetilde{A_{k}} \mathbf{f}\right)\left(x_{1}, \ldots, x_{n}\right)= & \frac{1}{\prod_{i=1}^{n}\left(\sigma\left(x_{i}\right)-a_{i}\right)} \\
& \times \int_{a_{1}}^{\sigma\left(x_{1}\right)} \ldots \int_{a_{n}}^{\sigma\left(x_{n}\right)} \widetilde{\mathbf{f}}\left(y_{1}, \ldots, y_{n}\right) \\
& \times \Delta y_{1} \cdots \Delta y_{n} .
\end{aligned}
$$

Proof. Statement follows from Theorem 2 using

$$
\begin{aligned}
& k\left(x_{1}, \ldots, x_{n}, y_{1}, \ldots, y_{n}\right) \\
& \quad=\left\{\begin{array}{ll}
1 & \text { if } a_{i} \leq y_{i}<\sigma\left(x_{i}\right) \leq b_{i}, \quad i \in\{1, \ldots, n\} \\
0 & \text { otherwise }
\end{array}\right\},
\end{aligned}
$$

since in this case

$$
\begin{aligned}
K\left(x_{1}, \ldots, x_{n}\right) & =\int_{a_{1}}^{\sigma\left(x_{1}\right)} \cdots \int_{a_{n}}^{\sigma\left(x_{n}\right)} \Delta y_{1} \cdots \Delta y_{n} \\
& =\prod_{i=1}^{n}\left(\sigma\left(x_{i}\right)-a_{i}\right) .
\end{aligned}
$$

Thus $A_{k}=\widetilde{A}_{k}, w=\widetilde{w}$. 
Corollary 13. If $a_{i}=0$ for all $i \in\{1, \ldots, n\}$ in $\mathbf{H} 4$ and $U \subset$ $\mathbb{R}^{m}$ is a closed convex set such that $\Phi: U \rightarrow \mathbb{R}$ is convex and continuous, then

$$
\begin{aligned}
& \int_{0}^{b_{1}} \ldots \int_{0}^{b_{n}} \Phi\left(\left(A_{k} \mathbf{f}\right)\left(x_{1}, \ldots, x_{n}\right)\right) \frac{\Delta x_{1} \cdots \Delta x_{n}}{x_{1} \cdots x_{n}} \\
& \quad \leq \int_{0}^{b_{1}} \cdots \int_{0}^{b_{n}} \prod_{i=1}^{n}\left(\frac{1}{y_{i}}-\frac{1}{b_{i}}\right) \Phi\left(\mathbf{f}\left(y_{1}, \ldots, y_{n}\right)\right) \Delta y_{1} \cdots \Delta y_{n}
\end{aligned}
$$

holds for all $\lambda_{\Delta}$-integrable $\mathbf{f}: \Lambda \rightarrow \mathbb{R}^{m}$ such that $\mathbf{f}(\Lambda) \subset U$, where

$$
\begin{aligned}
\left(A_{k} \mathbf{f}\right)\left(x_{1}, \ldots, x_{n}\right):= & \frac{1}{\prod_{i=1}^{n} \sigma\left(x_{i}\right)} \\
& \times \int_{0}^{\sigma\left(x_{1}\right)} \cdots \int_{0}^{\sigma\left(x_{n}\right)} \mathbf{f}\left(y_{1}, \ldots, y_{n}\right) \\
& \times \Delta y_{1} \cdots \Delta y_{n} .
\end{aligned}
$$

Proof. The statement follows from Theorem 12 using $\xi\left(x_{1}, \ldots, x_{n}\right)=1 / x_{1} \cdots x_{n}$, since in this case

$$
\begin{aligned}
w\left(y_{1}, \ldots, y_{n}\right) & =\int_{y_{1}}^{b_{1}} \cdots \int_{y_{n}}^{b_{n}} \frac{1}{\prod_{i=1}^{n} x_{i} \sigma\left(x_{i}\right)} \Delta x_{1} \cdots \Delta x_{n} \\
& =\prod_{i=1}^{n}\left(\frac{1}{y_{i}}-\frac{1}{b_{i}}\right) .
\end{aligned}
$$

Example 14. If $b_{i}=\infty$ for all $i \in\{1, \ldots, n\}$ in addition to the assumptions of Corollary 13, then (34) takes the form

$$
\begin{gathered}
\int_{0}^{\infty} \cdots \int_{0}^{\infty} \Phi\left(\left(A_{k} \mathbf{f}\right)\left(x_{1}, \ldots, x_{n}\right)\right) \frac{\Delta x_{1} \cdots \Delta x_{n}}{x_{1} \cdots x_{n}} \\
\leq \int_{0}^{\infty} \cdots \int_{0}^{\infty} \Phi\left(\mathbf{f}\left(y_{1}, \ldots, y_{n}\right)\right) \frac{\Delta y_{1} \cdots \Delta y_{n}}{y_{1} \cdots y_{n}} .
\end{gathered}
$$

Remark 15. Clearly, if the left-hand side is $\infty$ in (34), then right-hand side is also $\infty$.

Remark 16. For $\mathbb{T}=\mathbb{R}$ and $m=1$, inequality (37) is proved in $[4,24]$.

\section{Some Particular Cases}

In this section, firstly, we give Hilbert-type inequality on time scales.

Theorem 17. If $n=1, a_{1}=0$, and $b_{1}=\infty$ in $\mathbf{H} 4$ and for $q>1$ one defines

$$
\begin{gathered}
K_{1}(x)=\int_{0}^{\infty} \frac{(y / x)^{-1 / q}}{x+y} \Delta y, \\
K_{2}(y)=\int_{0}^{\infty} \frac{(y / x)^{1-1 / q}}{x+y} \Delta x,
\end{gathered}
$$

then

$$
\begin{gathered}
\int_{0}^{\infty}\left(\int_{0}^{\infty} \frac{(y / x)^{1-1 / q}\left(g_{1}(y)\right)^{p}}{x+y} \Delta y\right)^{1 / p} \\
\times\left(\int_{0}^{\infty} \frac{(y / x)^{1-1 / q}\left(g_{2}(y)\right)^{p^{\prime}}}{x+y} \Delta y\right)^{1 / p^{\prime}} \Delta x \\
\quad \geq \int_{0}^{\infty} K_{2}(y) g_{1}(y) g_{2}(y) \Delta y
\end{gathered}
$$

holds for all $\lambda_{\Delta}$-integrable $g_{j}: \Lambda \rightarrow \mathbb{R}_{+}$, where $j \in\{1,2\}$.

Proof. Use $\xi(x)=K_{1}(x) / x$ and $k(x, y)=$ $\left\{\begin{array}{c}(y / x)^{-1 / q} /(x+y), x \neq 0, y \neq 0, x+y \neq 0, \\ 0,\end{array}\right\}$ in Corollary 7 to obtain

$$
\begin{gathered}
\int_{0}^{\infty} \frac{K_{1}(x)}{x}\left(\frac{1}{K_{1}(x)} \int_{0}^{\infty} \frac{(y / x)^{-1 / q}\left(f_{1}(y)\right)^{p}}{x+y} \Delta y\right)^{1 / p} \\
\times\left(\frac{1}{K_{1}(x)} \int_{0}^{\infty} \frac{(y / x)^{-1 / q}\left(f_{2}(y)\right)^{p^{\prime}}}{x+y} \Delta y\right)^{1 / p^{\prime}} \Delta x \\
\geq \int_{0}^{\infty} w(y) f_{1}(y) f_{2}(y) \Delta y,
\end{gathered}
$$

where

$$
\begin{aligned}
w(y) & =\int_{0}^{\infty} \frac{k(x, y) \xi(x)}{K_{1}(x)} \Delta x=\int_{0}^{\infty} \frac{k(x, y)}{x} \Delta x \\
& =\frac{1}{y} \int_{0}^{\infty} \frac{(y / x)^{1-1 / q}}{x+y} \Delta x=\frac{K_{2}(y)}{y} .
\end{aligned}
$$

Using this value in (40), we obtain

$$
\begin{aligned}
& \int_{0}^{\infty}\left(\int_{0}^{\infty} \frac{(y / x)^{-1 / q}\left(f_{1}(y)\right)^{p}}{x+y} \Delta y\right)^{1 / p} \\
& \quad \times\left(\int_{0}^{\infty} \frac{(y / x)^{-1 / q}\left(f_{2}(y)\right)^{p^{\prime}}}{x+y} \Delta y\right)^{1 / p^{\prime}} \frac{\Delta x}{x} \\
& \geq \int_{0}^{\infty} K_{2}(y) f_{1}(y) f_{2}(y) \frac{\Delta y}{y} .
\end{aligned}
$$

Now, if we replace $f_{1}(y)$ with $g_{1}(y) y^{1 / p}$ and $f_{2}(y)$ with $g_{2}(y) \mathrm{y}^{1 / p^{\prime}}$, we obtain (39). 
Another Proof of (42). Consider L.H.S of (40) and applying Hölder inequality on time scale [25, Theorem 6.2] and Fubini theorem on time scale [16, Theorem 1.1], we have

$$
\begin{gathered}
\int_{0}^{\infty} \frac{K_{1}(x)}{x}\left(\frac{1}{K_{1}(x)} \int_{0}^{\infty} \frac{(y / x)^{-1 / q}\left(f_{1}(y)\right)^{p}}{x+y} \Delta y\right)^{1 / p} \\
\times\left(\frac{1}{K_{1}(x)} \int_{0}^{\infty} \frac{(y / x)^{-1 / q}\left(f_{2}(y)\right)^{p^{\prime}}}{x+y} \Delta y\right)^{1 / p^{\prime}} \Delta x \\
\geq \int_{0}^{\infty}\left(\int_{0}^{\infty} \frac{(y / x)^{-1 / q} f_{1}(y) f_{2}(y)}{x+y} \Delta y\right) \frac{\Delta x}{x} \\
\quad=\int_{0}^{\infty} f_{1}(y) f_{2}(y)\left(\int_{0}^{\infty} \frac{(y / x)^{-1 / q}}{x+y} \frac{\Delta x}{x}\right) \Delta y \\
\quad=\int_{0}^{\infty} \frac{f_{1}(y) f_{2}(y)}{y}\left(\int_{0}^{\infty} \frac{(y / x)^{1-1 / q}}{x+y} \Delta x\right) \Delta y \\
=\int_{0}^{\infty} K_{2}(y) f_{1}(y) f_{2}(y) \frac{\Delta y}{y} .
\end{gathered}
$$

Example 18. It is known that

$$
\int_{0}^{\infty} \frac{(y / x)^{-1 / q}}{x+y} d y=\int_{0}^{\infty} \frac{(y / x)^{1-1 / q}}{x+y} d x=\frac{\pi}{\sin (\pi / q)}
$$

for all $x, y \in \mathbb{R}_{+}=(0, \infty)$ with $q>1$. If $\mathbb{T}=\mathbb{R}$; then from (39), we obtain

$$
\begin{gathered}
\int_{0}^{\infty}\left(\int_{0}^{\infty} \frac{(y / x)^{1-1 / q}\left(f_{1}(y)\right)^{p}}{x+y} d y\right)^{1 / p} \\
\quad \times\left(\int_{0}^{\infty} \frac{(y / x)^{1-1 / q}\left(f_{2}(y)\right)^{p^{\prime}}}{x+y} d y\right)^{1 / p^{\prime}} d x \\
\quad \geq \frac{\pi}{\sin (\pi / q)} \int_{0}^{\infty} f_{1}(y) f_{2}(y) d y .
\end{gathered}
$$

In the rest of paper, we take $n=1, a \geq 0, b=\infty$ in $\mathbf{H 4}$.

Theorem 19. If (38) is satisfied, then

$$
\begin{gathered}
\int_{0}^{\infty}\left(\left(\int_{0}^{\infty} \frac{(y / x)^{1-1 / q}\left(f_{1}(y)\right)^{p}}{x+y} \Delta y\right)^{1 / p}\right. \\
\left.+\left(\int_{0}^{\infty} \frac{(y / x)^{1-1 / q}\left(f_{2}(y)\right)^{p}}{x+y} \Delta y\right)^{1 / p}\right)^{p} \Delta x \\
\geq \int_{0}^{\infty} K_{2}(y)\left(f_{1}(y)+f_{2}(y)\right)^{p} \Delta y
\end{gathered}
$$

Proof. Use $\xi(x)=K_{1}(x) / x$ and $k(x, y)=$ $\left\{\begin{array}{c}(y / x)^{-1 / q} /(x+y), x \neq 0, y \neq 0, x+y \neq 0, \\ 0, \quad \text { otherwise }\end{array}\right\}$ in Corollary 7 to obtain

$$
\begin{gathered}
\int_{0}^{\infty}\left(\left(\int_{0}^{\infty} \frac{(y / x)^{-1 / q}\left(f_{1}(y)\right)^{p}}{x+y} \Delta y\right)^{1 / p}\right. \\
\left.\quad+\left(\int_{0}^{\infty} \frac{(y / x)^{-1 / q}\left(f_{2}(y)\right)^{p}}{x+y} \Delta y\right)^{1 / p}\right)^{p} \frac{\Delta x}{x} \\
\geq \int_{0}^{\infty} K_{2}(y)\left(f_{1}(y)+f_{2}(y)\right)^{p} \frac{\Delta y}{y} .
\end{gathered}
$$

Now, if we replace $f_{1}(y)$ with $f_{1}(y) y^{1 / p}$ and $f_{2}(y)$ with $f_{2}(y) y^{1 / p}$, we obtain (46).

Remark 20. (a) We can give another proof of (47) using Minkowski's inequality on time scale [25, Theorem 7.2].

(b) If $p<1$, then we have reverse inequalities.

Now we consider some generalizations of the PólyaKnopp type inequalities.

Corollary 21. If (29) holds, and furthermore, if $U \subset \mathbb{R}^{m}$ is a closed convex set such that $\Phi: U \rightarrow \mathbb{R}$ is convex and continuous, then

$$
\begin{gathered}
\int_{a}^{\infty} \xi(x) \Phi\left(\frac{1}{\sigma(x)-a} \int_{a}^{\sigma(x)} \mathbf{f}(y) \Delta y\right) \Delta x \\
\leq \int_{a}^{\infty} w(y) \Phi(\mathbf{f}(y)) \Delta y
\end{gathered}
$$

holds for all $\lambda_{\Delta}$-integrable $\mathbf{f}: \Lambda \rightarrow \mathbb{R}^{m}$ such that $\mathbf{f}(\Lambda) \subset U$.

Proof. The statement follows from Theorem 12 using $n=1$.

Corollary 22. Assume (29). Then

$$
\begin{gathered}
\int_{a}^{\infty} \xi(x)\left(\frac{1}{\sigma(x)-a} \int_{a}^{\sigma(x)}\left(f_{1}(y)\right)^{p} \Delta y\right)^{1 / p} \\
\times\left(\frac{1}{\sigma(x)-a} \int_{a}^{\sigma(x)}\left(f_{1}(y)\right)^{p^{\prime}} \Delta y\right)^{1 / p^{\prime}} \Delta x \\
\geq \int_{a}^{\infty} w(y) f_{1}(y) f_{2}(y) \Delta y
\end{gathered}
$$

holds for all $\lambda_{\Delta}$-integrable $f_{j}: \Lambda \rightarrow \mathbb{R}_{+}$, where $j \in\{1,2\}$.

Proof. Statement follows from Corollary 7 using $m=2$. 
Corollary 23. Assume (29). Then

$$
\begin{gathered}
\int_{a}^{\infty} \xi(x)\left(\left(\frac{1}{\sigma(x)-a} \int_{a}^{\sigma(x)}\left(f_{1}(y)\right)^{p} \Delta y\right)^{1 / p}\right. \\
\left.\quad+\left(\frac{1}{\sigma(x)-a} \int_{a}^{\sigma(x)}\left(f_{2}(y)\right)^{p} \Delta y\right)^{1 / p}\right)^{p} \Delta x \\
\geq \int_{a}^{\infty} w(y)\left(f_{1}(y)+f_{2}(y)\right)^{p} \Delta y
\end{gathered}
$$

holds for all $\lambda_{\Delta}$-integrable $f_{j}: \Lambda \rightarrow \mathbb{R}_{+}$, where $j \in\{1,2\}$.

Proof. Statement follows from Corollary 8 using $m=2$.

Example 24. When $\mathbb{T}$ consists of isolated points, then from Corollary 22, we have

$$
\begin{aligned}
& \sum_{x \in[a, \infty)} \frac{\xi(x)(\sigma(x)-x)}{\sigma(x)-a} \\
& \quad \times\left(\sum_{y \in[a, \sigma(x))}\left(f_{1}(y)\right)^{p}(\sigma(y)-y)\right)^{1 / p} \\
& \times\left(\sum_{y \in[a, \sigma(x))}\left(f_{2}(y)\right)^{p^{\prime}}(\sigma(y)-y)\right)^{1 / p^{\prime}} \\
& \quad \geq \sum_{y \in[a, \infty)} w(y) f_{1}(y) f_{2}(y)(\sigma(y)-y),
\end{aligned}
$$

where $w(y)=\sum_{x \in[y, \infty)}(\xi(x)(\sigma(x)-x) /(\sigma(x)-a))$.

Example 25. When $\mathbb{T}$ consists of isolated points, then from Corollary 23, we have

$$
\begin{aligned}
& \sum_{x \in[a, \infty)} \frac{\xi(x)(\sigma(x)-x)}{\sigma(x)-a} \\
& \times\left(\left(\sum_{y \in[a, \sigma(x))}\left(f_{1}(y)\right)^{p}(\sigma(y)-y)\right)^{1 / p}\right. \\
& \left.\quad+\left(\sum_{y \in[a, \sigma(x))}\left(f_{2}(y)\right)^{p}(\sigma(y)-y)\right)^{1 / p}\right)^{p} \\
& \geq \sum_{y \in[a, \infty)} w(y)\left(f_{1}(y)+f_{2}(y)\right)^{p}(\sigma(y)-y),
\end{aligned}
$$

where $w(y)$ is the same as in Example 24 .
Example 26. For $\mathbb{T}=h \mathbb{N}=\{h n: n \in \mathbb{N}\}$ with $h>0, a=1$, and $\xi(x)=1 / \sigma(x),(51)$ takes the form

$$
\begin{gathered}
\sum_{n=1}^{\infty} \frac{1}{n(n+1)}\left(\sum_{k=1}^{n}\left(f_{1}(k h)\right)^{p}\right)^{1 / p} \\
\times\left(\sum_{k=1}^{n}\left(f_{2}(k h)\right)^{p^{\prime}}\right)^{1 / p^{\prime}} \\
\geq \sum_{n=1}^{\infty} \frac{1}{n} f_{1}(n h) f_{2}(n h) .
\end{gathered}
$$

Inequality (52) takes the form

$$
\begin{gathered}
\sum_{n=1}^{\infty} \frac{1}{n(n+1)}\left(\left(\sum_{k=1}^{n}\left(f_{1}(k h)\right)^{p}\right)^{1 / p}\right. \\
\left.+\left(\sum_{k=1}^{n}\left(f_{2}(k h)\right)^{p}\right)^{1 / p}\right)^{p} \\
\geq \sum_{n=1}^{\infty} \frac{1}{n}\left(f_{1}(n h)+f_{2}(n h)\right)^{p} .
\end{gathered}
$$

Example 27. For $\mathbb{T}=\mathbb{N}^{2}=\left\{n^{2}: n \in \mathbb{N}\right\}, a=1$, and

$$
\xi(x)=\frac{2(\sigma(x)-1)}{(\sigma(x)-x)^{2}(2 \sqrt{x}+3)},
$$

inequality (51) takes the form

$$
\begin{aligned}
& \sum_{n=1}^{\infty} \frac{2}{(2 n+1)(2 n+3)} \\
& \times\left(\sum_{k=1}^{n}(2 k+1)\left(f_{1}\left(k^{2}\right)\right)^{p}\right)^{1 / p} \\
& \times\left(\sum_{k=1}^{n}(2 k+1)\left(f_{2}\left(k^{2}\right)\right)^{p^{\prime}}\right)^{1 / p^{\prime}} \\
& \quad \geq \sum_{k=1}^{\infty} f_{1}\left(k^{2}\right) f_{2}\left(k^{2}\right) .
\end{aligned}
$$

Inequality (52) takes the form

$$
\begin{gathered}
\sum_{n=1}^{\infty} \frac{2}{(2 n+1)(2 n+3)} \\
\times\left(\left(\sum_{k=1}^{n}(2 k+1)\left(f_{1}\left(k^{2}\right)\right)^{p}\right)^{1 / p}\right. \\
\left.+\left(\sum_{k=1}^{n}(2 k+1)\left(f_{2}\left(k^{2}\right)\right)^{p}\right)^{1 / p}\right)^{p} \\
\geq \sum_{k=1}^{\infty}\left(f_{1}\left(k^{2}\right)+f_{2}\left(k^{2}\right)\right)^{p} .
\end{gathered}
$$


If we take

$$
\xi(x)=\frac{\sigma(x)-1}{x \sigma(x)},
$$

Inequality (51) takes the form

$$
\begin{gathered}
\sum_{n=1}^{\infty} \frac{(2 n+1)}{n^{2}(n+1)^{2}}\left(\sum_{k=1}^{n}(2 k+1)\left(f_{1}\left(k^{2}\right)\right)^{p}\right)^{1 / p} \\
\times\left(\sum_{k=1}^{n}(2 k+1)\left(f_{2}\left(k^{2}\right)\right)^{p^{\prime}}\right)^{1 / p^{\prime}} \\
\geq \sum_{k=1}^{\infty} \frac{(2 k+1)}{k^{2}} f_{1}\left(k^{2}\right) f_{2}\left(k^{2}\right) .
\end{gathered}
$$

Inequality (52) takes the form

$$
\begin{gathered}
\sum_{n=1}^{\infty} \frac{(2 n+1)}{n^{2}(n+1)^{2}} \\
\times\left(\left(\sum_{k=1}^{n}(2 k+1)\left(f_{1}\left(k^{2}\right)\right)^{p}\right)^{1 / p}\right. \\
\left.+\left(\sum_{k=1}^{n}(2 k+1)\left(f_{2}\left(k^{2}\right)\right)^{p}\right)^{1 / p}\right)^{p} \\
\geq \sum_{k=1}^{\infty} \frac{(2 k+1)}{k^{2}}\left(f_{1}\left(k^{2}\right)+f_{2}\left(k^{2}\right)\right)^{p} .
\end{gathered}
$$

By replacing $f_{1}\left(k^{2}\right)=\left(k^{2} /(2 k+1)\right)^{1 / p} f_{1}\left(k^{2}\right), f_{2}\left(k^{2}\right)=$ $\left(k^{2} /(2 k+1)\right)^{1 / \hat{p}} f_{2}\left(k^{2}\right)$ in (59) and in (60), we have

$$
\begin{gathered}
\sum_{n=1}^{\infty} \frac{(2 n+1)}{n^{2}(n+1)^{2}}\left(\sum_{k=1}^{n} k^{2}\left(f_{1}\left(k^{2}\right)\right)^{p}\right)^{1 / p} \\
\times\left(\sum_{k=1}^{n} k^{2}\left(f_{2}\left(k^{2}\right)\right)^{p^{\prime}}\right)^{1 / p^{\prime}} \\
\geq \sum_{k=1}^{\infty} f_{1}\left(k^{2}\right) f_{2}\left(k^{2}\right), \\
\sum_{n=1}^{\infty} \frac{(2 n+1)}{n^{2}(n+1)^{2}}\left(\left(\sum_{k=1}^{n} k^{2}\left(f_{1}\left(k^{2}\right)\right)^{p}\right)^{1 / p}\right. \\
\left.+\left(\sum_{k=1}^{n} k^{2}\left(f_{2}\left(k^{2}\right)\right)^{p}\right)^{1 / p}\right)^{p} \\
\geq \sum_{k=1}^{\infty}\left(f_{1}\left(k^{2}\right)+f_{2}\left(k^{2}\right)\right)^{p},
\end{gathered}
$$

respectively.
Example 28. For $\mathbb{T}=q^{\mathbb{N}}=\left\{q^{n}: n \in\{\mathbb{N}\}, q>1\right\}, a=q$ and

$$
\xi(x)=\frac{\sigma(x)-a}{\sigma(x)(\sigma(x)-x)},
$$

The inequality (51) takes the form

$$
\begin{aligned}
& \sum_{n=1}^{\infty} \frac{(q-1)}{q^{n}}\left(\sum_{k=1}^{n} q^{k-1}\left(f_{1}\left(q^{k}\right)\right)^{p}\right)^{1 / p} \\
& \quad \times\left(\sum_{k=1}^{n} q^{k-1}\left(f_{2}\left(q^{k}\right)\right)^{p^{\prime}}\right)^{1 / p^{\prime}} \geq \sum_{n=1}^{\infty} f_{1}\left(q^{n}\right) f_{2}\left(q^{n}\right) .
\end{aligned}
$$

Inequality (52) takes the form

$$
\begin{gathered}
\sum_{n=1}^{\infty} \frac{(q-1)}{q^{n}} \\
\times\left(\left(\sum_{k=1}^{n} q^{k-1}\left(f_{1}\left(q^{k}\right)\right)^{p}\right)^{1 / p}\right. \\
\left.+\left(\sum_{k=1}^{n} q^{k-1}\left(f_{2}\left(q^{k}\right)\right)^{p}\right)^{1 / p}\right)^{p} \\
\geq \sum_{n=1}^{\infty}\left(f_{1}\left(q^{n}\right)+f_{2}\left(q^{n}\right)\right)^{p} .
\end{gathered}
$$

Remark 29. (a) In classical case for $h=1$, inequalities (53) and (54) are the same as (1.7) and (1.9), also (63) and (64) are the same as (1.6) and (1.8) in [18, Corollary 1.3], respectively, while according to authors knowledge, (56), (57), (59), (60), and (61) are not existing in the literature.

(b) For $p<1$, we get the reverse inequalities.

Remark 30. The results given in Section 5 can analogously be proved for $\Omega=\Lambda=\left[a_{1}, \infty\right)_{\mathbb{T}} \times \cdots \times\left[a_{n}, \infty\right)_{\mathbb{T}}$.

Remark 31. The results given in Corollaries 7 and 8 and in their given applications can also be obtained for finite value of $m>2$ analogously.

\section{Acknowledgments}

The research of the first author is supported by a grant of the Romanian National Authority for Scientific Research, CNCS UEFISCDI, project no. PN-II-ID-PCE-2011-3-0154. The research of the second author is partially supported by the Higher Education Commission, Pakistan, and the research of the third author is supported by the Croatian Ministry of Science, Education and Sports under the research Grant 1171170889-0888.

\section{References}

[1] G. H. Hardy, J. E. Littlewood, and G. Pólya, Inequalities, Cambridge Mathematical Library, Cambridge University Press, Cambridge, UK, 1988, Reprint of the 1952 edition. 
[2] E. K. Godunova, "Inequalities based on convex functions," Izvestija Vysših Učebnyh Zavedeniǔ Matematika, vol. 1965, no. 4, pp. 45-53, 1965.

[3] S. Kaijser, L. Nikolova, L.-E. Persson, and A. Wedestig, "Hardytype inequalities via convexity," Mathematical Inequalities \& Applications, vol. 8, no. 3, pp. 403-417, 2005.

[4] S. Kaijser, L.-E. Persson, and A. Öberg, "On Carleman and Knopp's inequalities," Journal of Approximation Theory, vol. 117, no. 1, pp. 140-151, 2002.

[5] K. Krulić, J. Pečarić, and L.-E. Persson, "Some new Hardy type inequalities with general kernels," Mathematical Inequalities \& Applications, vol. 12, no. 3, pp. 473-485, 2009.

[6] D. S. Mitrinović, J. E. Pečarić, and A. M. Fink, Inequalities Involving Functions and Their Integrals and Derivatives, vol. 53 of Mathematics and its Applications, Kluwer Academic, Dordrecht, The Netherlands, 1991.

[7] U. M. Ozkan and H. Yildirim, "Hardy-Knopp-type inequalities on time scales," Dynamic Systems and Applications, vol. 17, no. 3-4, pp. 477-486, 2008.

[8] U. M. Özkan and H. Yıldırım, "Time scale Hardy-Knopp type integral inequalities," Communications in Mathematical Analysis, vol. 6, no. 1, pp. 36-41, 2009.

[9] P. Řehák, "Hardy inequality on time scales and its application to half-linear dynamic equations," Journal of Inequalities and Applications, no. 5, pp. 495-507, 2005.

[10] M. Bohner, A. Nosheen, J. E. Pečarić, and A. Younus, "Some dynamic Hardy-type inequalities with general kernel," Journal of Mathematical Inequalities. In press.

[11] M. Bohner and A. Peterson, Dynamic Equations on Time Scales, Birkhäuser, Boston, Mass, USA, 2001.

[12] M. Bohner and G. Sh. Guseinov, "Multiple integration on time scales," Dynamic Systems and Applications, vol. 14, no. 3-4, pp. 579-606, 2005.

[13] M. Bohner and G. Sh. Guseinov, "Multiple Lebesgue integration on time scales," Advances in Difference Equations, vol. 2006, Article ID 26391, 12 pages, 2006.

[14] G. Sh. Guseinov, "Integration on time scales," Journal of Mathematical Analysis and Applications, vol. 285, no. 1, pp. 107-127, 2003.

[15] H. L. Royden, Real Analysis, Macmillan Publishing Company, New York, NY, USA, 3rd edition, 1988.

[16] R. Bibi, M. Bohner, J. E. Pečarić, and S. Vorošanec, "Minkoski and Beckenback-Oresher inequalities and functionals on time scales," Journal of Mathematical Inequalities. In press.

[17] M. Anwar, R. Bibi, M. Bohner, and J. E. Pečarić, "Jensen’s functional on timescales for multi-variables," submitted.

[18] P. M. Vasić and J. E. Pečarić, "Notes on some inequalities for convex functions," Matematički Vesnik, vol. 6, no. 19, pp. 185193, 1982

[19] E. Beck, "Über Ungleichungen von der Form $f\left(M_{\varphi}(x ; \alpha)\right.$, $\left.M_{\psi}(y ; \alpha)\right) \geqq M_{\chi}(f(x, y) ; \alpha)$," Univerzitet u Beogradu. Publikacije Elektrotehničkog Fakulteta. Serija Matematika i Fizika, vol. 320-328, pp. 1-14, 1970.

[20] D. S. Mitrinović, J. E. Pečarić, and A. M. Fink, Classical and New Inequalities in Analysis, Kluwer Academic, 1993.

[21] L. Horváth, Kh. Ali Khan, and J. Pečarić, "Refinements of Hölder and Minkowski inequalities with weights," Proceedings of A. Razmadze Mathematical Institute, vol. 158, pp. 33-56, 2012.

[22] L. Horváth, K. A. Khan, and J. E. Pečarić, "Refinements of Hölder and Minkowski inequalities with weights," Proceedings of A. Razmadze Mathematical Institute, vol. 158, pp. 33-56, 2012.
[23] M. Krnić, N. Lovričević, and J. E. Pečarić, "On the properties of McShane's functional and their applications," Periodica Mathematica Hungarica, vol. 66, no. 2, pp. 159-180, 2013.

[24] R. P. Boas, Jr. and C. O. Imoru, "Elementary convolution inequalities," SIAM Journal on Mathematical Analysis, vol. 6, pp. 457-471, 1975.

[25] M. Anwar, R. Bibi, M. Bohner, and J. Pečarić, "Integral inequalities on time scales via the theory of isotonic linear functionals," Abstract and Applied Analysis, vol. 2011, Article ID 483595, 16 pages, 2011. 


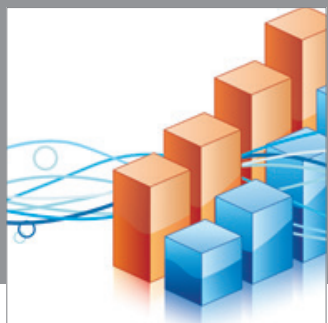

Advances in

Operations Research

mansans

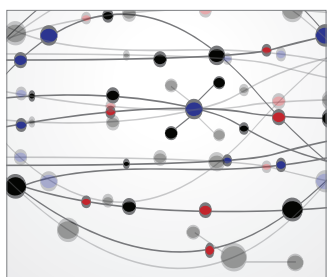

The Scientific World Journal
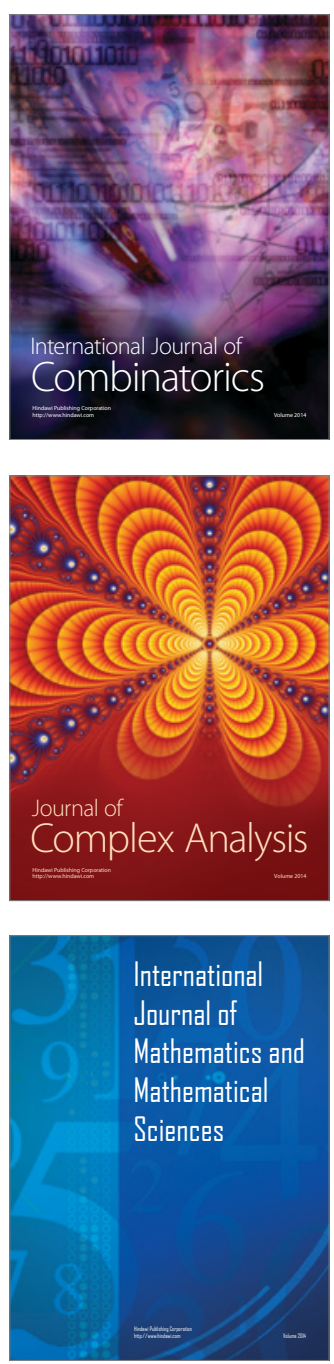
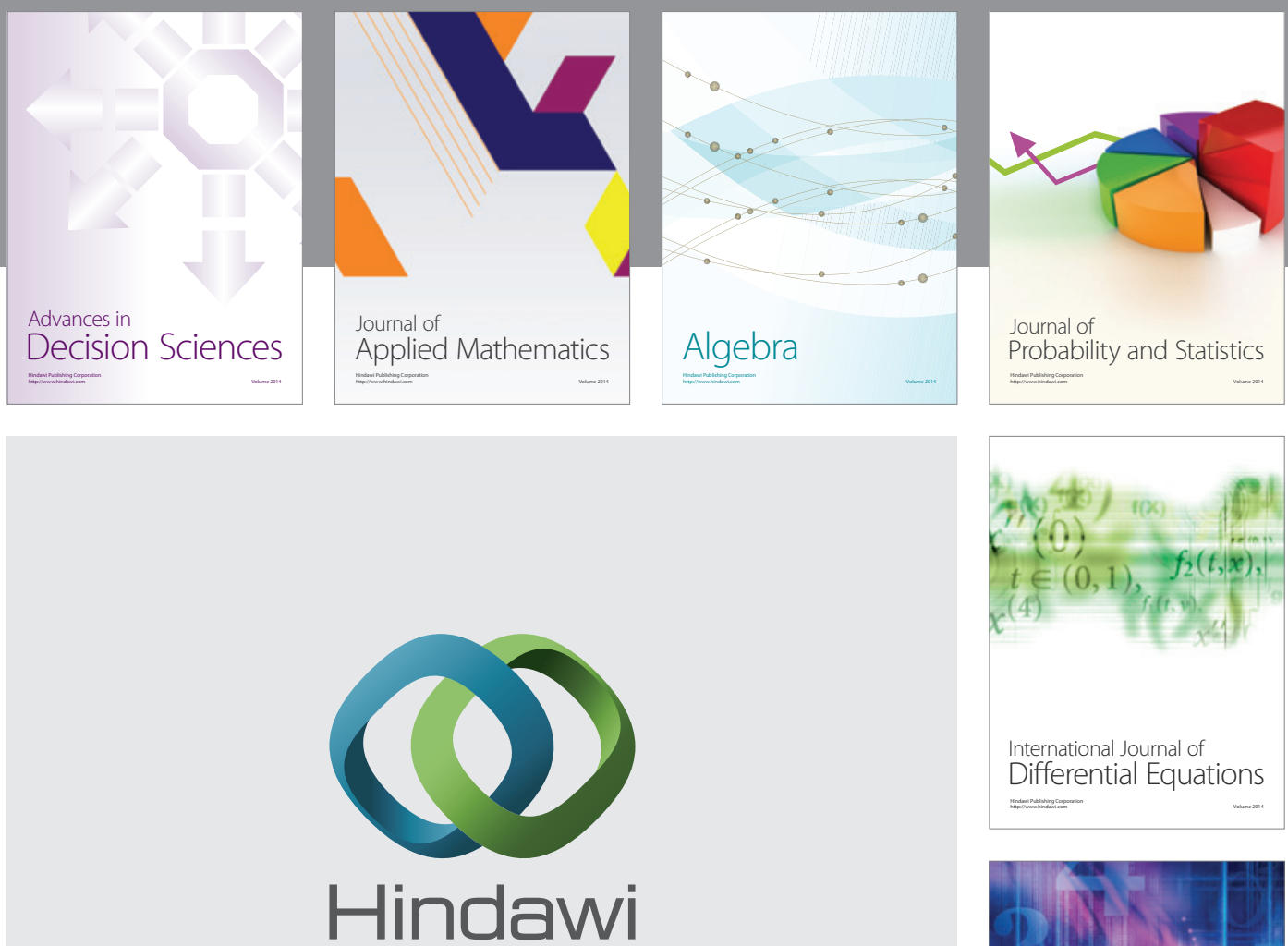

Submit your manuscripts at http://www.hindawi.com
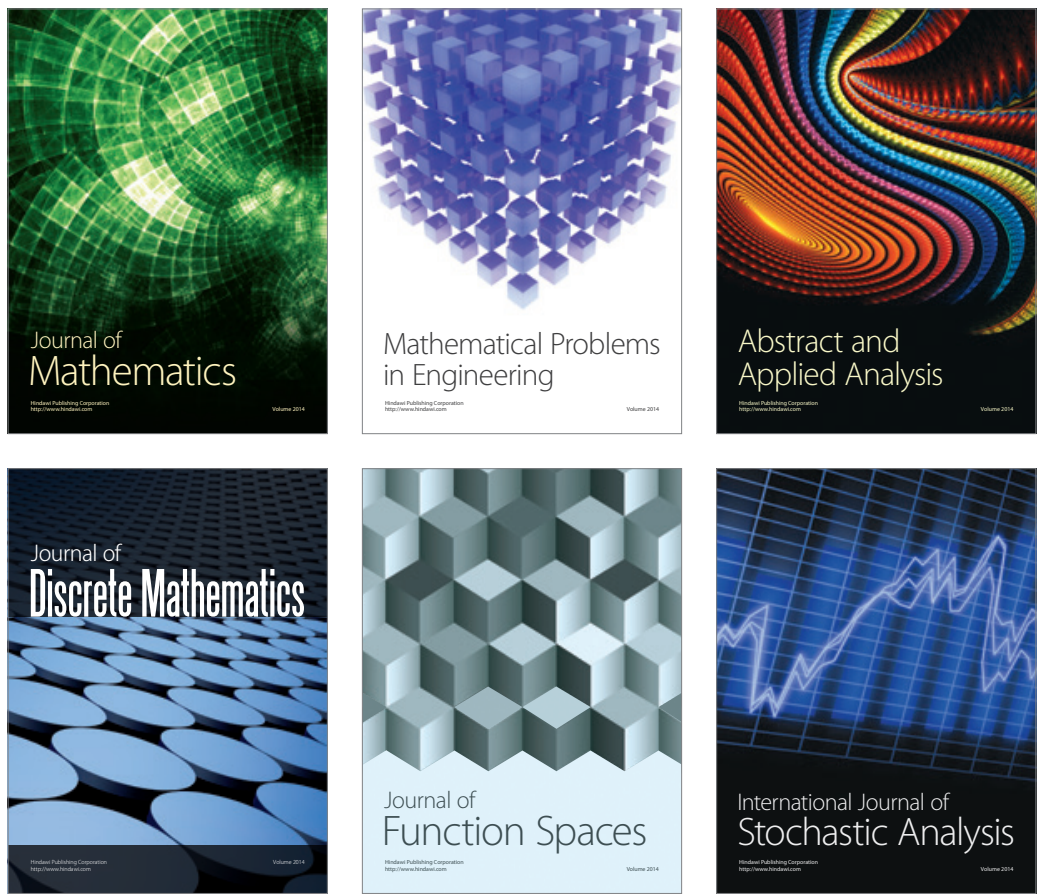

Journal of

Function Spaces

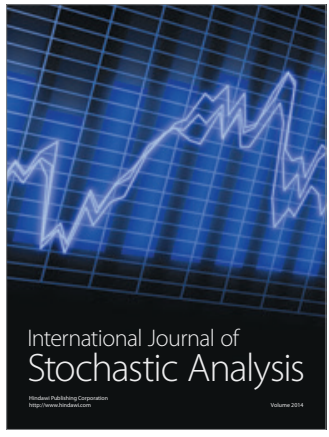

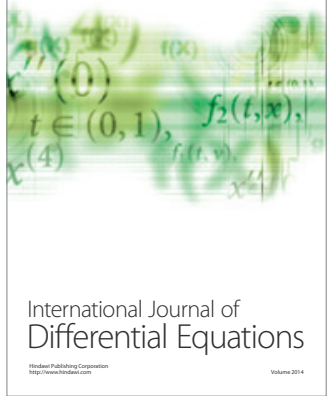
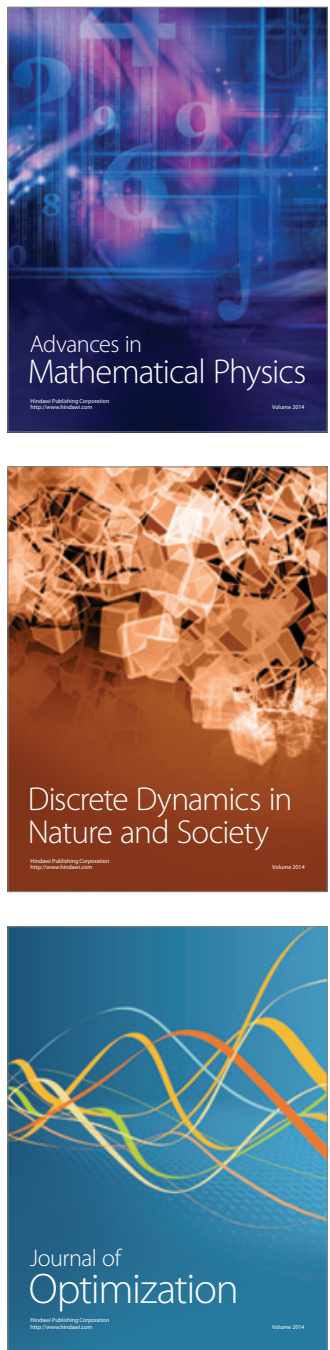